\title{
Toward Principles for Visual Interaction Design for Communicating Weight by using Pseudo-Haptic Feedback
}

\author{
Kumiyo Nakakoji \\ Key Technology Laboratory \\ SRA Inc. \\ 2-32-8 Minami-lkebukuro, Toshima, \\ Tokyo, 171-8513, Japan \\ kumiyo@sra.co.jp
}

\author{
Yasuhiro Yamamoto \\ Precision and Intelligence Laboratories \\ Tokyo Institute of Technology \\ 4259 Nagatsuda, Midori, \\ Yokohama, 226-8503, Japan \\ yxy@acm.org
}

\author{
Yasuharu Koike \\ Precision and Intelligence Laboratories \\ Tokyo Institute of Technology \\ 4259 Nagatsuda, Midori, \\ Yokohama, 226-8503, Japan \\ koike@pi.titech.ac.jp
}

\begin{abstract}
The goal of our research is to communicate "weight" by using the pseudo-haptic feedback, without actually generating force to a user's hand, thereby not requiring actuators. Our approach is to use EMG signals as an input device, and to identify a set of principles for visual interaction design to provide an illusion of stiffness and viscosity.
\end{abstract}

pseudo-haptics, illusion, visual interaction design

\section{INTRODUCTION}

Pseudo-haptics is a phenomenon where visuo-haptic sensory conflicts and the sensory dominance of vision over touch cause a haptic illusion when perceiving spatial properties (Lecuyer 2009). That is, one is likely to perceive a haptic sensation that is different from the haptic property present in the real world when appropriate visual feedback is provided. For instance, one feels a bump on a computer display when a mouse cursor on the screen becomes slowing down with respect to the physical mouse movement (Lecuyer, Burkhardt, Etiennne 2004). Researchers have found through user studies that humans perceive pseudo-haptics in terms of object stiffness (Lecuyer, Coquillart, Kheddar, 2000), texture (Lecuyer, Burkhardt, Etiennne 2004), mass (Dominjon et al. 2005), and hand-displacement (Pusch, Martin, Coquillart 2008).

Our approach is to use pseudo-haptics in the design of human-computer interaction. The goal of our current project is to communicate "weight," or mass, by using the pseudo-haptic feedback, without actually generating force to a user's hand, and thereby not requiring actuators. We use EMG signals as an input device (Koike, Yamamoto, Nakakoji 2006), trying to identify a set of principles for visual interaction design to provide an illusion of stiffness and viscosity.

\section{PSEUDO-HAPTICS IN HCI}

Pseudo-haptics occurs when visual properties and tactile properties captured through the sensor channels exhibit an inconsistency, or a conflict, in terms of the model of the world a person expects to perceive. Since visual properties are dominant over tactile properties, the person perceives tactile properties different than the actual physical properties so that the perceived visual and tactile properties produce a coherent view of the world.

Lecuyer (2009) provides a detailed survey on existing studies on what pseudo haptics is, how it is accounted, and the application of pseudo-haptic feedback in human-computer interaction for virtual environments.

In one of their early experimental studies on pseudo haptics on texture (Lecuyer, Burkhardt, Etiennne 2004), they displayed the mouse cursor on a screen, which moved in accordance with a mouse device operated by a study participant (just like a regular mouse and a mouse cursor). The study participant 
was instructed to move a mouse cursor from left to right to go across the screen display moving over a coloured area on the display. They covered the study participant's hand operating a mouse with a box so that the participant could not visually monitor the hand operating the mouse. When the visual cursor reached an edge of the coloured area, they decreased the motion speed of the cursor. Thus, the cursor suddenly slowed down in the coloured area despite that the participant kept moving the mouse in the same speed. Then, the participant reported that they felt the bumps with the hand. In the follow-up study, they changed the size of the mouse cursor in addition to decreasing the motion speed, and found the even stronger effects (Lecuyer, Burkhardt, Tan 2008).

Thus, using pseudo-haptic feedback makes it possible "to stimulate haptic sensations, such as stiffness or friction, without necessarily using a haptic interface" (Lecuyer 2009). Following the scheme, the goal of our project is to stimulate haptic sensation of "weight" for a user, without using actuators attached to a person's forearms.

\section{INDUCING ILLUSIONS OF WEIGHT}

Our approach can be viewed as a way to exploring the illusion of weight.

An illusion is a distortion of the senses (WIKIPEDIA), where the information gathered by the senses is processed by the brain to give a percept that is not in correspondence with the actual physical properties of the stimulus source.

Illusions of colour are probably most familiar to us. As an example, we perceive the colour orange when yellow stripes are put over the pink area surrounded by the thick blue border, while we perceive the colour purple when blue stripes are put over the same pink area surrounded by the yellow area (Figure 1; reproduced based on (Color Illusion 12)).

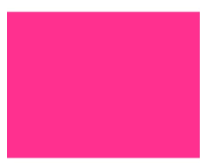

$(\mathrm{R}=255, \mathrm{G}=0, \mathrm{~B}=127)$

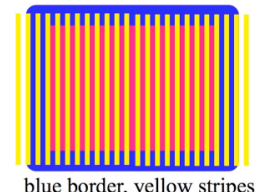

blue border, yellow stripes

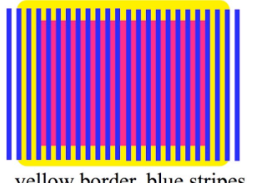

yellow border, blue stripes
Figure 1: A Colour Illusion Example (reproduced based on (Color Illusion 12))

A size-weight illusion is a tactile illusion where a larger object is generally perceived lighter than a smaller object if they have the same weight. Ross has found that the perceived weight of an object is a linear function of the logarithm of its density when only the volume is changed and weight being constant (Ross 1969). The study has also found that the material of an object affects the expectation of how it weighs, and tins are perceived slightly heavier than polystyrene blocks (Ross 1969).

Although an illusion is "an erroneous perception of reality" (American Heritage Dictionary), it is based on an "adjustment" made by the brain during perception, and commonly shared by most people. Illusions are "errors committed by the brain rather than by the senses" (Goldstein 1999 as cited in Lecuyer 2009).

On one hand, illusion can be misleading and has been something to pay an attention to in human-computer interaction. For instance, people might make a mistake in interpreting information in coloured graphs as a result of a colour illusion. On the other hand, illusion can be something to take an advantage of in interaction design. For instance, with the above colour illusion example, we do not need to use orange in order to make people perceive orange; by appropriately placing the colours pink, blue and yellow, we could produce either the "orange" or "purple" effect.

Our approach in the design of human computer interaction is following the latter; to take an advantage of the existence of such illusions as a property of human perception system, something to nurture.

\section{FOUNDATION: TELE-KINESTHETIC INTERACTION ENVIRONMENT}

In one of our previous projects, we have developed a "tele-kinesthetic" environment, where a user remotely interacts with a tangible $3 \mathrm{D}$ object display using EMG (Figure 2) (Koike, Yamamoto, Nakakoji 2006). The tele-kinesthetic interaction environment consists of two components: MyKinSynthesizer and PhyMotion.

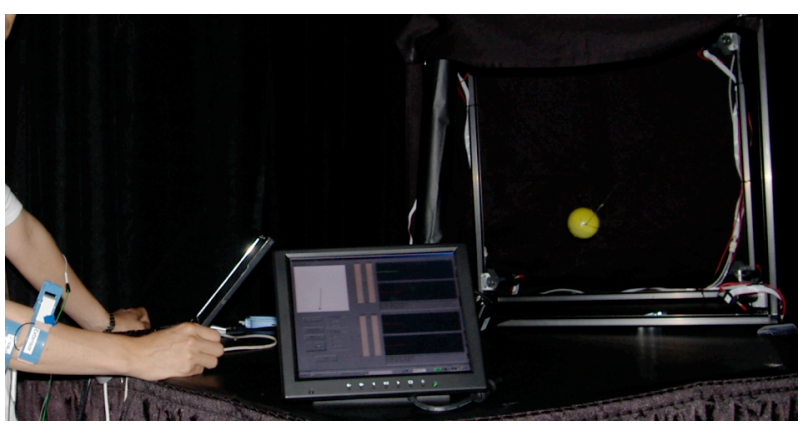

Figure 2: Tele-Kinesthetic Interaction Environment 
MyKinSynthesizer collects EMG signals via EMG electrodes attached on the user's forearm. Different parts of different muscles produce distinctive patterns of signals as the user moves and strains the hand. We have developed an algorithm to synthesize such different sources of signals into a musculo-skeletal model. We use the musculo-skeletal model to dynamically calculate the current torque, stiffness, joint angle and posture of the user's hand, and to approximate the motion and the force of the user's hand.

PhyMotion is an extension of SPIDAR, a 3D input and haptic feedback device, developed by Sato et al. (Ishii, Sato 1994). We have extended SPIDAR into PhyMotion as a programmable 3D tangible motion display. The position and the movement of the ball are now controlled by PhyMotion through eight strings attached to the eight corners of the cubic frame. We can program the behaviour of the ball by controlling the strings as if it obeys the law of physics.

The tele-kinesthetic interaction environment feeds the 3D motion and force data of the user's hand calculated by MyKinSynthesizer into PhyMotion. When the user moves and strains the hand, the motion of the ball of PhyMotion is updated by taking into account the user's hand's motion and force data, making the user feel as if he/she is engaged in a tele-kinesthetic interaction with the ball of PhyMotion.

\section{THE MODEL OF WEIGHT-ILLUSION}

Lecuyer (2009) describes steps to design a pseudo-haptic system that simulates a given haptic property (p.51): (1) to identify a law that controls that haptic property and associates it with spatial parameters; (2) to set up a visuo-haptic sensory conflict focusing on a spatial parameter associated with this haptic property; and (3) to modify the perception of the targeted haptic property and create pseudo-haptic feedback by simply modifying the visual feedback of this spatial parameter.

In modifying the visual feedback, the notion called C/D (Control/Display) ratio is introduced (Lecuyer, Burkhardt, Etiennne 2004), which refers to how to change the speed of hand movement (Control) in relation to the speed of cursor movement (Display).

Following the scheme, we have developed a model to stimulate haptic sensation of "weight" for a user. Based on our previous work on the tele-kinesthetic interaction environment, we use EMG as a haptic input device, and virtual display, 3-D object display, or robots as a visual feedback media.
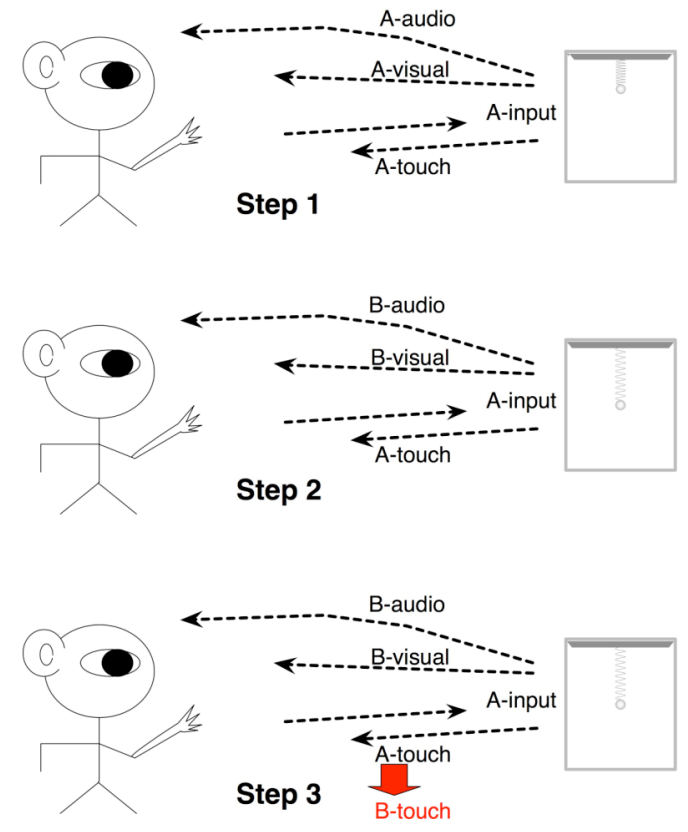

Figure 3: A Proposed Model for a Weight Illusion

Figure 3 illustrates the model of how to stimulate the weight illusion.

(i)Step 1: A person interacts with a displayed object using EMG of his or her forearm. When he or she is putting force $(A)$ on the hand, the object moves and produces visual (and auditory, when applicable) feedback to his or her sensory system. Here, the mapping between the touch and visual (and auditory) senses are established (i.e., A-touch $=$ A-visual $=$ A-audio) within his or her brain.

(ii)Step 2: The C/D (Control/Display) ratio is then changed so that the visual (and auditory) feedback would be slightly different $(B)$ despite that the force put on the hand remain the same $(A)$. Now, the person's brain identifies a sensory conflict (i.e., A-touch != B-visual and B-audio).

(iii)Step 3: Because of the visual (and auditory) perceptual dominance over touch, the person perceives B-touch, instead of A-touch.

\section{ELEMENTS TO STUDY}

In order to identify a set of visual interaction design principles for an environment to communicate 
weight by using the pseudo-haptic feedback mechanism, we have started our project by focusing on the following research questions to be experimentally studied.

(i) How long (or how many trials) it takes for a person to establish the mapping between the haptic property and the visual and auditory properties in Step 1? Or would it even be necessary?

(ii) How long (or how many trials) it takes for the person to sense the conflict between the haptic property and the visual and auditory properties in Step 2?

(iii)What should be the $C / D$ (Control/Display) ratio for different types of visual feedback for a person to perceive the targeted weight? How about the C/D ratio for the audio display?

(iv)Would there be differences among different media for visual feedback, such as virtual display, a tangible 3D object, or robots?

(v)Would there be differences among different types of visual representations in virtual display as feedback, such as a robot-arm, pendulum, spring balance, or elastic band?

(vi)Would there be differences among different viewpoints and perspectives of visual representations as feedback? For instance, how a robot arm is positioned in relation to a person's posture at which height; facing to each other or placing it side-by-side?

(vii)What would be the effects of feedback delay in stimulating weight-illusion?

(viii)Would it be possible to keep stimulating the weight illusion in a consistent manner over a long period of time?

Most of existing studies on pseudo haptics focus on the identification of pseudo haptic perceptions of physical properties. We are not aware of any research that focuses on the identification of design principles for an interactive multimodal environment that makes use of this peculiar nature of human perceptive mechanism.

Existing studies mostly depend on self-reporting of study participants (such as through questionnaire) to analyze whether and how pseudo haptic perceptions take place. We additionally measure EMG to examine whether and how pseudo haptic weight is perceived by people.

Regarding the first question, existing studies primarily ask study participants to either compare the two conditions, or to identify the condition that exhibits the closest spatial property. We have not found any studies that reported the effect of the length and style of this initial mapping period.

Moreover, this initial mapping process may not be necessary in the case of weight illusion of a physical object. In our previous study where we measured hand trajectory and surface electromyography to estimate time-varying stiffness and torque patterns in joints of a person engaging in a task of loading a 3D object on the hand, we have found that the participants not only produced force with cocontraction and increasing stiffness levels according to the weight of the object, but also exhibited different levels of stiffness according to the "expected" weight of an object even before holding it, thereby indicating that stiffness can be an effective parameter as an efferent signal for weight perception (Koike, Kim, Duk 2006). We as humans learn how physical objects weigh through our own experience interacting with the real world. We know from our experience that a thick book weighs heavy, and a thin brochure weighs light. And we a priori control the stiffness of our hand before holding them accordingly.

Regarding the last question, although this is our premise for the project, it has still been under study whether pseudo-haptics can really be regarded as sensory-illusion. Pseudo-haptic feedback may not be related to perceptual characteristics (which is an inevitable process) but could be "a strategic decision-making process" related to "the experiment itself and could correspond to the learning of a systematic association of sensorimotor displacement and visual feedback" (which is reversible) (Lecuyer 2009).

\section{IWE: ILLUSORY WEIGHT EXPLORATORIUM}

To study the experimental factors listed above, we have developed a study environment called IWE (Illusory Weight Exploratorium). In IWE, a user (i.e., an experimenter) can choose a visual representation for a visual feedback, and interactively changes the parameters for the orientation and perspective of the visual display, 


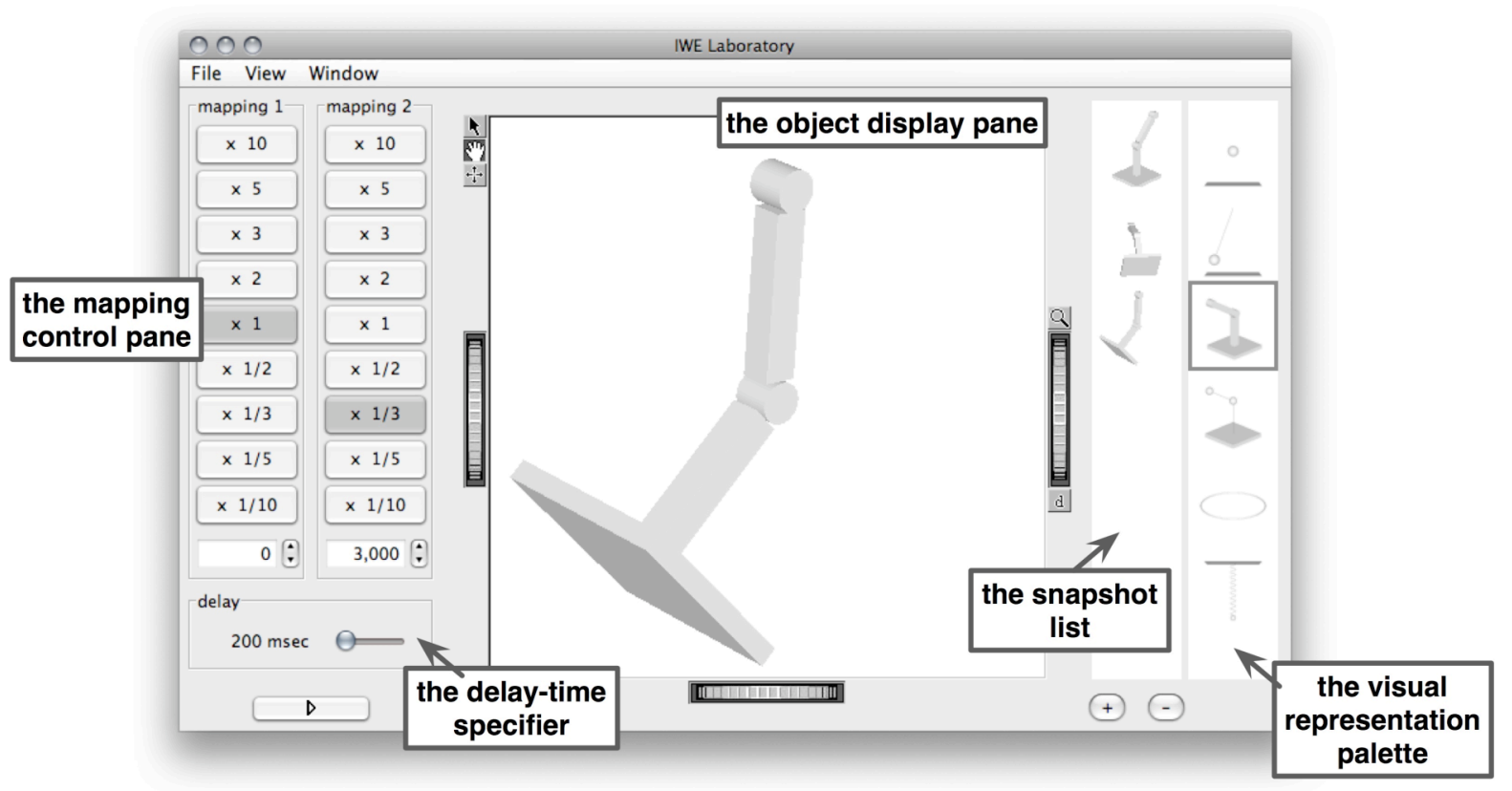

Figure 4: IWE: Illusory Weight Exploratorium

C/D ratio, and the time duration for the displays. The user may take a snapshot of the visual orientation and perspective setting for each visual display for later retrieval.

IWE consists of five parts: (1) the object display pane, (2) the visual representation palette, (3) the snapshot list, (4) the two mapping control panes, and (5) the delay-time specifier (Figure 4).

When a user chooses one of the visual displays from the visual representation palette, the 3D object is displayed in the object pane. The pane is equipped with the zoomer, perspective changer, and object rotator dials. The user may adjust the orientation, the perspective, and the size of the object displayed in the object display pane and takes a snapshot of the current visual setting by clicking on the "+" button located in the bottom of the snapshot list. Clicking on one of the snapshot thumbnails listed in the snapshot list brings back the visual setting of the state when the snapshot was taken.

Each 3D object moves its movable part according to the input signal (which comes from EMG). For instance, the robot arm shown in the figure moves its forearm in the range of 180 degrees. The elastic band and the spring balance shrink and stretch, the pendulum moves its ball.
With the mapping control pane, the user maps the input signal to the visual object movement; that is, to change the C/D (Control/Display) ratio. The user can create two mappings, mapping 1 for the Step 1 in Figure 3, for a study participant to establish the mapping, and mapping 2 for Step 2 in Figure 3, for the participant to perceive the conflict stimulating a weight illusion. The user can currently set the C/D ratio in the range from $\times 0.1$ to $\times 10$. The user specifies the time-duration for engaging in each of the mapping in the window in the bottom of each of the mapping control pane in the unit of millisecond.

Finally, the user may controls the delay time for visual feedback to occur in terms of the input signal with the delay-time specifier. If it sets to 200 milliseconds, the visual display movement is reflecting the signal it received 200 milliseconds ago.

For an actual experiment, the object display pane is spawned from IWE as an individual window so that a study participant may only look at the object display pane on a separate monitor while the experimenter controls the settings with the original IWE. We are currently designing a set of user experiments using IWE to investigate the research questions listed above.

\section{DISCUSSIONS}


The research contribution of this work would be twofold.

First, it demonstrates an innovative interaction method, where users would be able to communicate weight and mass without wearing actuators to physically generate force to their hands. We are currently working on the wide varieties of the application of the interaction method. For instance, we may apply the technique as a way to give a feedback to a doctor engaging in remote operation. The force put on the knife of a remote operation robot can be communicated with the doctor through visual and auditory feedback. As another example, one may communicate how a product under design weighs with remote team members in a distributed design meeting. As a third example, the weight should not necessarily be that of a physical object, but could be associated with a conceptual property. Programming component that has significant impact on other components could be assigned heavy weight so that a programmer may perceive the weight of importance of the component when editing it.

Second, the use of pseudo-haptics opens up a large area of research where the design of dynamically changing inter-modal relations might more effectively communicate information with users in multi-modal environments. Multi-modal environments have tried to enforce more immersive, more realistic feedback, such as through organic user interfaces, where input equals output (Vertegaal, Poupyrev 2008). The use of illusion, such as the pseudo-haptic feedback, however, makes us consider how a user perceives the world through multiple sensory channels; we may need to alternate information on some of the channels so that the user would perceive the world more effectively. The notion of "direct manipulation" in $\mathrm{HCl}$, thus, may need to be re-contextualized.

\section{ACKNOWLEDGEMENTS}

This work was supported by JST, CREST.

\section{REFERENCES:}

Color Illusion

12 http://www.psy.ritsumei.ac.jp/ akitaoka/color12e.ht ml (06 May 2010)

Dominjon, L., Lecuyer, A., Burkhardt, J.M., Richard, PI, Richir, S. (2005) Influence of control/display ratio on perception of mass of manipulated objects in virtual environments, IEEE Virtual Reality 2005 (VR'05), pp.19-25.

Ishii, M., Sato, M. (1994) A 3D Spatial Interface Device Using Tensed Strings, Presence, Vol.3, No.1, pp.81-86.
Koike, Y., Kim, J., Duk, S. (2006) Role of Stiffness in Weight Perception, Japanese Psychological Research, Volume 48 Issue 3, pp.174-187.

Koike, Y., Yamamoto, Y., Nakakoji, K. (2006) Tele-Kinesthetic Interaction: Using Hand Muscles to Interact With a Tangible 3D Object, SIGGRAPH2006, Emerging Technologies, Boston, MA..

Lecuyer, A., Coquillart, S., Kheddar, A., Richard, P., Coiffet, P. (2000) Pseudo-haptic Feedback: Can Isometric Input Devices Simulate Force Feedback?, Proceedings of the IEEE International Conference on Virtual Reality.

Lecuyer, A., Burkhardt, J.M., Etiennne, L. (2004) Feeling Bumps and Holes Without a Haptic Interface, The Perception of Pseudo-Haptic Textures, Proceedings of $\mathrm{CHI} 2004$, Vienna, Austria, pp.239-246.

Lecuyer, A., Burkhardt, J.M., Tan, C.H. (2008) A Study of the Modification of the Speed and Size of the Cursor for Simulating Pseudo-Haptic Bumps and Holes, ACM Transactions on Applied Perception, No.5, Vol.3, Article 14, pp.1-21.

Lecuyer, A. (2009) Simulating Haptic Feedback Using Vision: A Survey of Research and Applications of Pseudo-Haptic Feedback, Presence: Teleoperators and Virtual Environments, Vol.18, No.1, pp.39-53, MIT Press.

Pusch, A., Martin, O., Coquillart, S. (2008) HEMP: Hand-Displacement-Based Pseudo-Haptics: A Study of a Force Field Application, Proceedings of IEEE Symposium on 3D User Interfaces, pp.50-66.

Ross, H.E. (1969) When is a Weight Not Illusory?, The Quarterly Journal of Experimental Psychology, Volume 21, Issue 4, pp.346-355.

Vertegaal, R., Poupyrev, I. (2008) Organic User Interfaces: Introduction to Special Issue, Communications of the ACM 51(6), pp.26-30.

WIKIPEDIA, http://en.wikipedia.org/wiki/Illusion (06 May 2010) 